[Agr. Biol. Chem., Vol. 36, No. 4, p. 695 696, 1972]

\title{
Increase in Silk Production by the Silkworm, Bombyx mori L., due to Oral Administration of a Juvenile Hormone Analog
}

Sir:

Till recently, it was generally considered that the practical utilization of juvenile hormones and related compounds would be developed in the area of insect control due to the disruptions of physiological and biochemical systems specific to insects. In a preceeding paper, "however, we reported that fifth instar larvae of the silkworm, Bombyx mori L., to which some 3,4-methylenedioxyphenyl derivatives structurally related to Cecropia juvenile hormones were topically applied, produced giant cocoons. We suggested that this procedure could be utilized to increase the silk productivity of the insect. Subsequently, we examined the effect of a juvenile hormone analog from this series on silk production using oral administration.

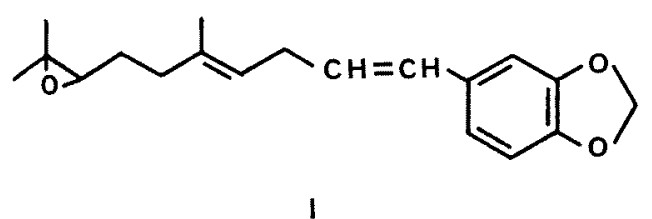

Fifth instar larvae of the silkworm were reared on an artificial diet, which was prepared by mixing mulberry leaf powder $\left(50.00_{0}\right)$, defatted soybean meal $\left(24.00^{\circ}\right)$, cellulose powder $(15.0 \%)$, agar powder $(5.0 \%)$, citric acid $(4.0,0)$, L-ascorbic acid (1.5\%) and sorbic acid $(0.5 \%)$ with distilled water $(2.5 \mathrm{ml} / \mathrm{g}$ solid) followed by steaming at $80 \sim 85^{\circ} \mathrm{C}$ for $15 \mathrm{~min}$. The juvenile hormone analog, 1-(3, 4-methylenedioxyphenyl)-5,9-dimethyl-8-epoxy-1,4-decadiene (I), composed of stereoisomers, was used solely throughout this experiment because of its accessibility through synthetic preparation ${ }^{21}$ and its favorable biological effect. ${ }^{11}$ A given amount of the reagent was dissolved in a small amount of acetone, then mixed with diet components before adding water. Ten larvae of the hybrid $8.3 \times 3.4$ were used for each dose and were kept at $25 \sim 27^{\circ} \mathrm{C}$. The body weight of each larva was measured $144 \mathrm{hr}$ after the fourth molt. A weight measurement was made for each cocoon and the cocoon-layer taken from it after complete spinning.

In a preliminary trial, we fed the reagent for $48 \mathrm{hr}$ in dietary doses of $0.5,1.0$ and 2.0 ppm to fifth instar larvae of various growth stages to find the best time for administration. Early stage feeding (during the $48 \mathrm{hr}$ just after the fourth molt) was almost ineffective for increasing cocoon weight, even at a dosage of $2.0 \mathrm{ppm}$. However, middle stage feeding (during the $48 \mathrm{th} \sim 96 \mathrm{th} \mathrm{hr}$ of the instar) prolonged duration of the larval stage, which resulted in increases in both the cocoon and cocoon-layer weights. This efficacy became distinct as the dose increased.

We, then, investigated the effect of the compound at higher doses $(2.0,4.0$ and 8.0 ppm) at the middle stage of the instar. Typical results are illustrated in Table I. A dose of $4.0 \mathrm{ppm}$ had the most favorable effects. At this level, the instar was prolonged 66.4 hr ( $147.6 \mathrm{hr}$ with the control), resulting in an increase in the cocoon-layer weight 1.41 times that of the control. Administration of 8.0 ppm produced heavier cocoons, but the ratio 
Table I. Growth and Metamorphosis in Fifth Instar larvae of the SILKWORM ORALLY ADMINISTERED COMPOUND I

The same nubmers of males and females were used for each dose. The compound was fed during the $48 \mathrm{th} \sim 96 \mathrm{th} \mathrm{hr}$ of the instar.

\begin{tabular}{ccccccc}
$\begin{array}{c}\text { Content of } \\
\text { compd. in } \\
\text { diet, ppm }\end{array}$ & $\begin{array}{c}\text { Initial } \\
\text { larval wt. } \\
\mathrm{g}\end{array}$ & $\begin{array}{c}\text { Larval wt. } \\
\text { 144 hr after } \\
4 \text { th molt, } \mathrm{g}\end{array}$ & $\begin{array}{c}\text { Length of } \\
\text { 5th instar, } \\
\text { hr }\end{array}$ & $\begin{array}{c}\text { Wt. of } \\
\text { cocoon, } \\
\mathrm{g}\end{array}$ & $\begin{array}{c}\text { Wt. of } \\
\text { cocoon- } \\
\text { layer, }\end{array}$ & $\begin{array}{c}\text { Ratio of } \\
\text { cocoon- } \\
\text { layer, } \%\end{array}$ \\
\hline 0 & 0.620 & 3.219 & 147.6 & 1.423 & 0.283 & 19.9 \\
2 & 0.650 & 3.882 & 193.3 & 1.787 & 0.385 & 21.5 \\
4 & 0.650 & 3.900 & 214.0 & 1.920 & 0.398 & 20.7 \\
8 & 0.645 & 4.025 & 225.0 & 2.050 & 0.380 & 18.5 \\
\hline
\end{tabular}

of cocoon-layer weight to cocoon weight was smaller than that of the control.

We, also, examined the efficacy of the compound, when it was fed for $24 \mathrm{hr}$ at $2.0,4.0$ or $8.0 \mathrm{ppm}$ in the diet. In general, administration during the $48 \mathrm{th} \sim 72 \mathrm{nd}, 72 \mathrm{nd} \sim 96 \mathrm{th}$ or $96 \mathrm{th} \sim 120 \mathrm{th} \mathrm{hr}$ of the final instar induced significant increases in cocoon-layer weights varying $20 \sim 35 \%$ with the dose tested. Feeding at $8.0 \mathrm{ppm}$ during the $72 \mathrm{nd} \sim 96 \mathrm{th} \mathrm{hr}$ was the most effective. Administration at a later stage (during the $120 \mathrm{th} \sim 140 \mathrm{th} \mathrm{hr}$ ) revealed hardly any promotive effect even at $8.0 \mathrm{ppm}$.

Thus, we have demonstrated that productivity of silk can be enhanced through oral administration of juvenile hormones to fifth instar larvae of the silkworm, though the yield was not as remarkable as that with topical application. ${ }^{11}$

\section{REFERENCES}

1) C.-F. Chang, S. Murakoshi and S. Tamura, Agr. Biol. Chem., 36, 692 (1972).

2) C.-F. Chang and S. Tamura, ibid., 35, 1307 (1971).

Shigeo MURaKosHI

Ching-Fun CHANG*

Saburo TAMURA ${ }^{* \dagger}$

Kanagawa Prefectural Institute for

Sericultural Science

Ebina-shi, Kanagawa-ken

* Department of Agricultural Chemistry

The University of Tokyo

Bunkyo-ku, Tokyo

Received January 31, 1972

$\uparrow$ To whom all correspondence should be addressed. 